\title{
Implementation of recommendations from the Philippine Academy of Rehabilitation Medicine's Stroke Rehabilitation Guideline: a plan of action
}

This article was published in the following Dove Press journal:

Clinical Audit

30 August 2013

Number of times this article has been viewed

\author{
Consuelo B Gonzalez- \\ Suarez ${ }^{1-3}$ \\ Janine Margarita R Dizon ${ }^{3}$ \\ Karen Grimmer ${ }^{4}$ \\ Myrna S Estrada ${ }^{2,5}$ \\ Edgardo D Uyehara ${ }^{2,6}$ \\ Lauren Anne S Liao ${ }^{1,2}$ \\ Anne-Rochelle D Malleta ${ }^{2,7}$ \\ Ma Elena R Tan ${ }^{2,6}$ \\ Vero Marfil ${ }^{6}$ \\ Cristine R Sanchez-Versales ${ }^{\prime}$ \\ Jimah L Suarez ${ }^{2,7}$ \\ Kleon C So' \\ Philip John B Fabia' \\ Jose Bonifacio S Rafanan ${ }^{2,8}$ \\ Romil M Martinez ${ }^{2,9}$ \\ 'University of Santo Tomas Hospital, \\ Manila, Philippines; ${ }^{2}$ Philippine \\ Academy of Rehabilitation Medicine, \\ Quezon City, Philippines; ${ }^{3}$ University \\ of Santo Tomas, Manila, Philippines; \\ ${ }^{4}$ International Centre for Allied Health \\ Evidence, University of South Australia \\ Adelaide, SA, Australia; ${ }^{5}$ Dela Salle \\ University Hospital, Cavite, Philippines; \\ 'Veterans' Memorial Medical Center. \\ Quezon City, Philippines; ${ }^{7}$ Philippine \\ Orthopedic Center, Quezon City, \\ Philippines; ${ }^{8}$ Philippine General \\ Hospital, Manila, Philippines; ${ }^{9}$ Amang \\ Rodriguez Memorial Medical Center, \\ Rizal, Philippines
}

Correspondence: Consuelo B GonzalezSuarez

University of Santo Tomas Hospital,

Espaňa St, Manila, Philippines 1500

Tel +6327245730

Fax +6327409713

Email bebetsuarez6I@gmail.com
Background: Based on the best current evidence, stroke rehabilitation significantly improves health outcomes. The Philippine Academy of Rehabilitation Medicine (PARM) recently developed Filipino contextualized guidelines for stroke rehabilitation. This paper presents a protocol for the implementation phase of the Stroke Rehabilitation Guideline.

Methods: A multidisciplinary working group comprising medical and paramedical personnel engaged in stroke rehabilitation in four Filipino training hospitals attended a 2-day workshop to develop an implementation plan for six high-evidenced recommendations for inpatient rehabilitation. Recommendations were for early referral for rehabilitation, increasing intensity of exercise, dysphagia assessment, pressure sore assessment and treatment, and a planned rehabilitation discharge summary.

Results: A three-step protocol was developed for guideline implementation: (1) designing an audit protocol to describe current stroke care practice; (2) identifying the most appropriate methods for effectively disseminating the guideline; and (3) considering the best approaches to change professional behaviors relating to the implementation strategies, including these local Filipino professional training, practice and service provision models. Clinical protocols were developed to support standard application of the recommendations.

Conclusion: It is anticipated that by applying effective guideline dissemination strategies adapted for local contexts and addressing specific stakeholder learning and behavior change requirements, the potential for sustained uptake of the guidelines will be achieved. This will result in less stakeholder resistance to evidence-based practices, better processes and health outcomes, fewer adverse events, and more effective resource utilization.

Keywords: stroke rehabilitation, evidence-based practice, clinical practice guidelines, implementation, behavior change

\section{Background}

Stroke usually affects an individual's mobility and performance of activities in daily living. Rehabilitation based on the current best evidence has been shown to significantly improve function and is underpinned by the timely provision of effective services aimed in assisting an individual to resume usual activities to the best possible capacity. ${ }^{1,2}$ About 486 strokes per 100,000 persons occur annually in the Philippines. ${ }^{3}$ The Philippines is a developing country in which equitable access to evidence-based rehabilitation for stroke patients is a challenge for a range of environmental, personal, organizational, and educational reasons. ${ }^{4,5}$ For instance, a culture of evidence-based practice is not widespread, and education on evidence-based practice is not broadly available to health care providers. ${ }^{6}$ In addition, not every Filipino has access to specialist 
medical personnel for reasons of geography and workforce availability. $^{5}$

We recently published the first stage of our guideline project which was based on the contextualization of Western clinical guidelines for stroke and low back pain for conditions in the Philippines. ${ }^{7}$ Prior to this work, there was no nationally agreed guideline to guide Filipino best practice for stroke rehabilitation. We took a novel approach by contextualizing relevant guidelines because there was no gain in "reinventing the wheel," given the prohibitive costs of developing de novo guidelines. Further, we believed that our limited resources should be focused on three areas: (1) making current highquality evidence-based recommendations relevant to the local Philippine context; (2) ensuring these would be accessible to, and useful for, health care providers; and (3) implementing the guidelines effectively and sustainably.

This paper presents the next stage of the project: the design of the implementation phase of the Stroke Rehabilitation Guideline $^{14}$ using Michie et al's framework ${ }^{8}$ within the quality improvement steps described by Grol and Grimshaw. ${ }^{9}$ Implementation plans should be informed by multiple sources of data and multiple strategies. Thus, we took a three-step approach in designing the implementation plans: ${ }^{10-12}$

1. creating a description of current practice against which changes could be compared and potentially attributed to guideline implementation;

2. determining the best method(s) of disseminating guidelines to address the needs of stakeholders within their local contexts of practice;

3. outlining the most appropriate strategies to use to change professional behavior within the context of local service provision.

The three key issues which required consideration in the implementation of the stroke guidelines were: (1) ways of disseminating guidelines to stakeholders using interventions which were most likely to change behaviors, (2) the outcomes which could best be measured before and after guideline dissemination, and (3) the expected practice behavior changes from guideline implementation.

\section{Methods}

\section{Ethics}

Ethical approval was obtained from the Institutional Review Board of the University of Santo Tomas Hospital, Manila, Philippines.

\section{Working group}

A multidisciplinary working group (WG) was formed with seven physiatrists (rehabilitation medicine specialists), two physiotherapists, and two occupational therapists who were engaged in stroke rehabilitation. They were from key training hospitals. An external international facilitator (KG) participated, providing educational input and independent evaluation and critique of the WG's activities.

\section{Stakeholders}

The WG identified primary and secondary stakeholders in the Stroke Rehabilitation Guideline. The primary stakeholders were the target users (physiatrists, physical and occupational therapists, speech pathologists, nurses), as they were responsible for rehabilitation decisions and activities. Secondary stakeholders consisted of other medical specialists who were engaged in stabilizing acute stroke patients prior to commencement of rehabilitation, and patients and their families who were the recipients of rehabilitation, and who had input into preferred treatment options and compliance.

\section{Recommendations targeted for implementation}

The Filipino contextualized stroke guidelines contained 307 recommendations, which were mapped to a "typical" Filipino patient journey. They were then endorsed by Philippine Academy of Rehabilitation Medicine (PARM) using wording which reflected the strength of the underlying evidence base. ${ }^{7}$ For example, PARM strongly endorsed that stroke patients should be mobilized as early as possible - within 24 hours after onset of symptoms, unless medically contraindicated, because there was a strong level of evidence for this recommendation. Following the principles for guideline implementation, not all recommendations were targeted for inclusion in the implementation plan. ${ }^{13}$ Six recommendations were chosen for this purpose, which were selected because they had the

Table I Recommendations to be used in the implementation plan

I. Early referral to stroke patient rehabilitation unit once medically stable

2. Rehabilitation should be increased according to the tolerance of the patient as it has beneficial effects on functional outcome, including gait

3. The typical swallow screening procedure should be performed prior to removal of nasogastric tube

4. Pressure care risk assessment should be undertaken followed by regular regular evaluation of the skin for the possible development of pressure sores

5. To prevent pressure sores, appropriate pressure-relieving aids and strategies should be provided, such as a pressure-relieving mattress, use of proper positioning, turning, and transferring techniques and judicious use of barrier sprays, lubricants, and protective dressings to avoid skin injury due to maceration, friction, or excessive pressure

6. On discharge, patients should be referred to an outpatient stroke rehabilitation clinic and into the care of an organized rehabilitation team

Note: data obtained from the Stroke Rehabilitation Guideline. ${ }^{14}$ 
strongest evidence supporting their effectiveness in inpatient stroke rehabilitation; these are presented in Table $1 .{ }^{14}$

\section{Developing the implementation plan}

The implementation plan was developed over one intensive weekend in Manila in November 2012. Day 1 commenced with education sessions presented by KG, CBG-S, and JMRD on the principles of evidence-based practice, implementation science, behavior change, and auditing. KG presented information on Australian experiences of implementing clinical practice guidelines. After presentation of a number of behavior change models, the core elements of Michie et al's behavior change wheel - capabilities, opportunity and motivation, or "COM-B" system, were endorsed by the WG as the most appropriate framework for implementation and behavior change. ${ }^{8}$

The WG then broke into smaller, discipline-specific, working groups which debated:

- how current stroke rehabilitation practice could be described in the Philippines;

- specific needs of different stakeholder groups in terms of dissemination of the recommendations;

- likely behaviors which would need to be targeted via dissemination strategies to improve uptake of the guideline;

- ways in which change in practice could be demonstrated.

To guide discussions, a list of dissemination strategies and their estimates of effect was compiled via literature review. ${ }^{15-21}$ How these strategies might influence behaviors of primary and secondary stakeholders were considered by the small groups (in terms of the elements of Michie et al's behavior wheel ${ }^{8}$ ).

A list of items related to the six chosen recommendations was then developed by the small groups, which could be used for auditing purposes.

On Day 2, the collated findings from the Day 1 discussions were provided to the whole WG. After reflection and discussion, modifications were made to the audit items and dissemination strategies.

The WG then developed evidence-based protocols which provided details to assist health care providers to implement the six recommendations. The $\mathrm{WG}$ believed that standard descriptors of assessments and measures, interventions, and timeframes for interventions, would assist in increasing guideline compliance, and underpinning future standard recording of audit items. ${ }^{22,23}$ The WG used targeted literature searches to identify the most recent and highest hierarchy evidence, which provided details to populate practice protocols using the PARM writing guide ${ }^{14}$ on how to grade literature. ${ }^{7}$ The WG looked at work published by quality and safety committees in other countries, particularly by those in the UK, the USA, and Australia, as well as work published by individual researchers. ${ }^{22-24}$

The initial stage of the planned audit was then discussed. This would establish current practice in treating stroke patients in the Philippines. To date, as far as we are aware, there is still no information on current Filipino practices in stroke rehabilitation. A range of issues underpinning the collection of representative and accurate data on Filipino stroke rehabilitation practices was discussed, relevant to the inaugural current practice audit and future audits of the same data, to assess practice change.

\section{Results}

\section{Understanding current practice}

The WG agreed that the Stroke Rehabilitation Guideline had been developed on the basis of common presentations to Filipino physiatrists, rather than evidence of poor practice. The WG identified that, prior to guideline implementation, a description of current practices was required, which could be used to underpin guideline implementation strategies. Re-auditing many of these measures could be used to establish guideline compliance after implementation. Description of current practices (baseline) and re-evaluation of current practice (post-implementation) are elements of quality improvement cycles. ${ }^{9}$ Initial audit measures to understand current practice are outlined in Table 2. The last two (grey) columns relate to measures which, on re-audit, could indicate change that could be attributed to the implementation of clinical guidelines. These items are discussed later in the "Results" section.

\section{Implementation strategies}

The WG identified a range of strategies which members believed would be required for effective dissemination of guideline information to stakeholders within the context of required behavior change. The strategies are presented below and are summarized in Table 3.

\section{Physiatrists}

- Handouts or pamphlets on the key recommendations of the guidelines should be provided to every member of PARM and all the training institutions.

- Regular education sessions on evidence based practice (EBP) and outcome measures and updates on guidelines.

- Progress reports on guideline roll out.

\section{Other medical specialists}

- Promotion of rehabilitation medicine services: 


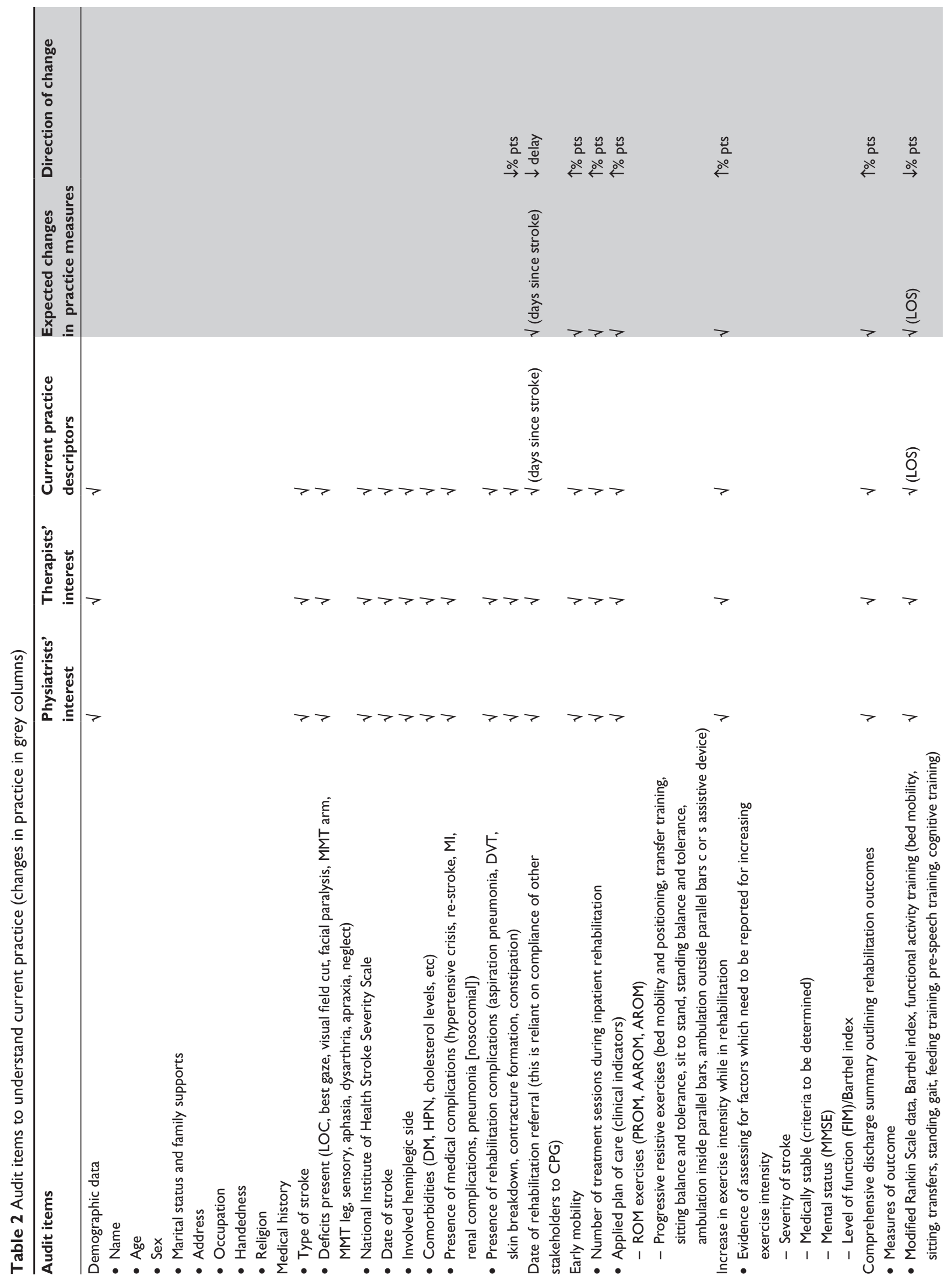



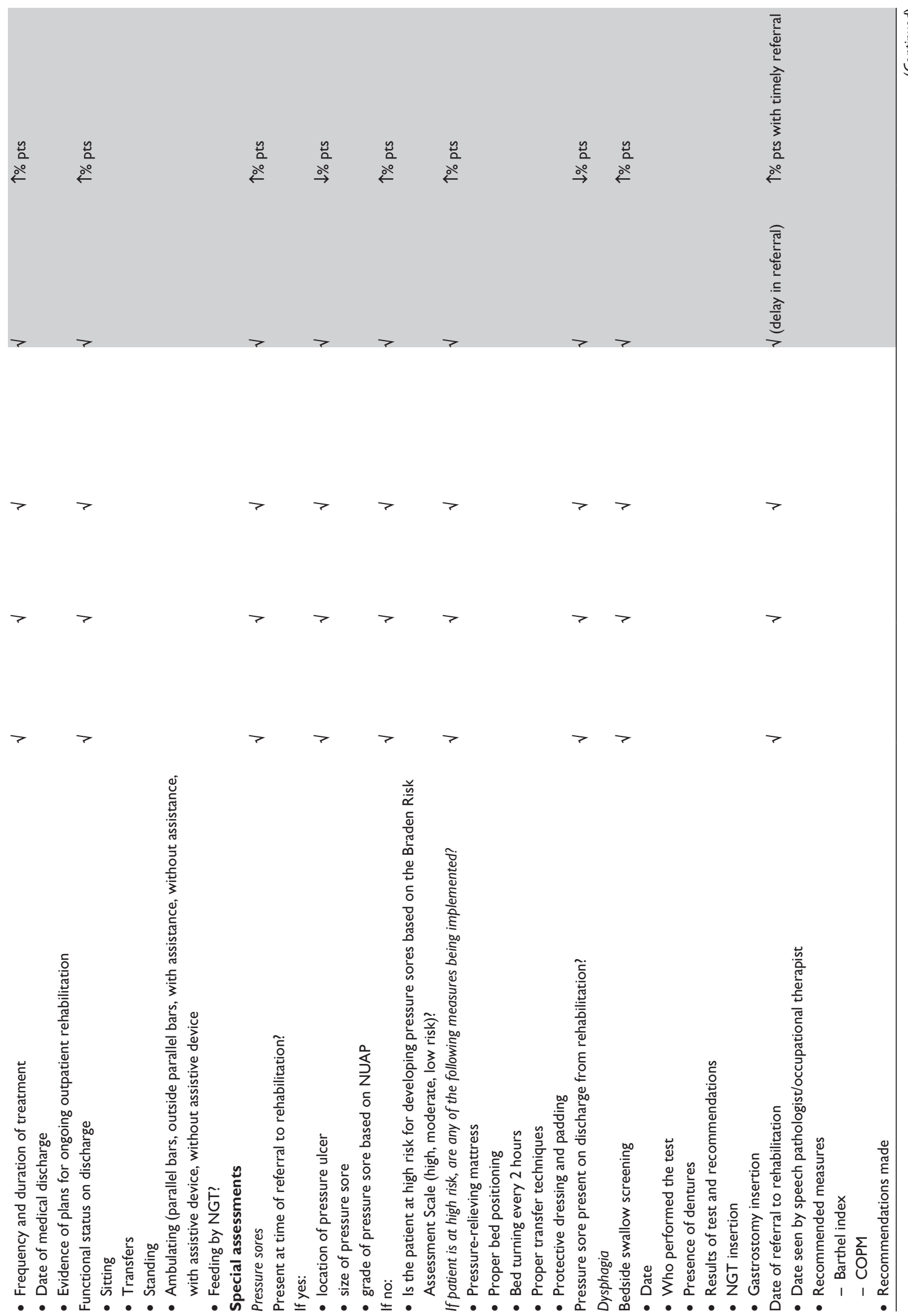


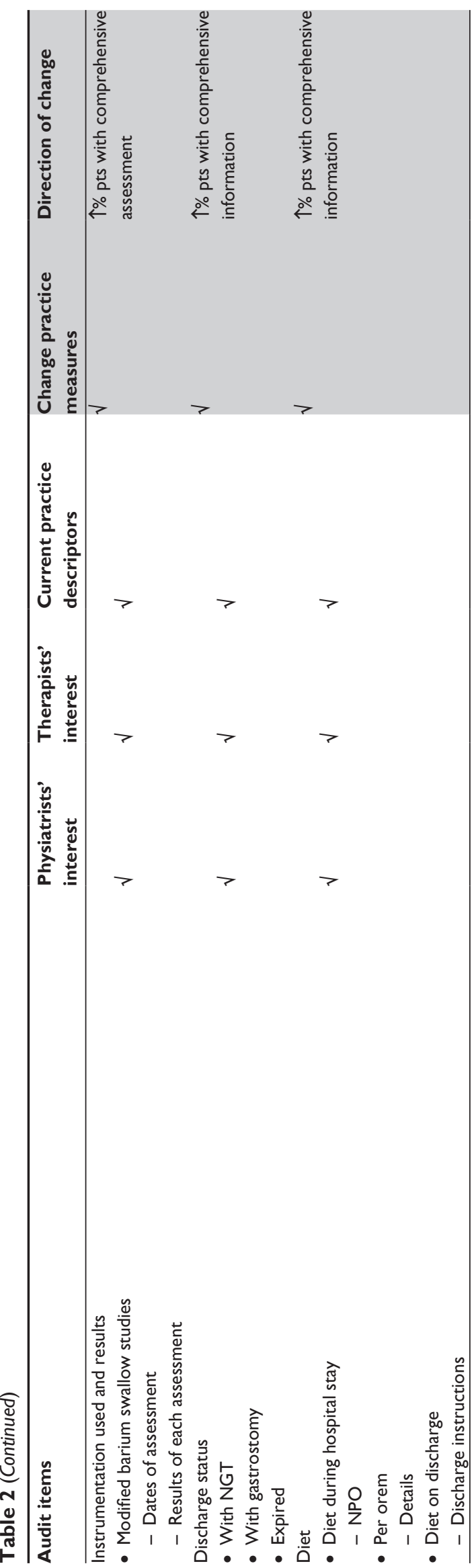

- education on rehabilitation should be provided at training institutions;

- agreement with administrative heads of clinical departments (internal medicine, cardiology, neurology) should be obtained regarding the importance of guideline-driven acute stroke care, and rehabilitation;

- education on early intervention rehabilitation.

- Indications and contraindications

- Comorbidities management such as dysphagia, pressure sore, bladder incontinence, deep venous thrombosis

- integrated process flowchart (see Table S1)

- stroke rehabilitation checklist

- Pressure sore assessment

- Dysphagia assessment tool

\section{Physical and occupational therapists}

- Small-group discussions regarding the guidelines ensuring that therapists were actively involved

- Educational training

- Mini case presentations

- Involvement in actual protocols or demonstrations

- Quick guides (posters, forms, summaries)

- Identifying senior staff who may be resistant to innovative changes and engaging them in local activities

- Opinion leaders

\section{Nurses}

- Training provided on proper bed mobility techniques and transfers

- Provision of educational training guides

- Opinion leaders

\section{Patients}

- Targeted patient education (importance of rehabilitation, adherence to exercises)

- Quick guides (posters, forms, summaries)

- Demonstration of exercises/activities with return demonstration from the patient and relative

- Visual aids or handouts on exercises

- Lectures focusing on the benefits of post-stroke rehabilitation

\section{Behavior change}

Table 4 outlines the WG's considerations of the relationships between the proposed dissemination strategies and the COM-B elements of Michie et al's behavior change wheel. ${ }^{8}$ This table highlights the importance of multiple strategies 
Table 3 Summarized dissemination strategies

\begin{tabular}{|c|c|c|c|c|c|}
\hline & Physiatrists & Other doctors & Therapists & Nurses & Patients \\
\hline Handouts/pamphlets/quick guides & $\sqrt{ }$ & $\sqrt{ }$ & $\sqrt{ }$ & $\sqrt{ }$ & $\sqrt{ }$ \\
\hline Lectures providing education about guidelines & $\sqrt{ }$ & $\sqrt{ }$ & $\sqrt{ }$ & & \\
\hline Lectures about early rehabilitation & & $\sqrt{ }$ & $\sqrt{ }$ & $\sqrt{ }$ & $\sqrt{ }$ \\
\hline Demonstrations & & & & $\sqrt{ }$ & $\sqrt{ }$ \\
\hline Progress reports on guideline roll out & $\sqrt{ }$ & & & & \\
\hline Promotion of rehabilitation medicine as a specialty by networking & & $\sqrt{ }$ & & & \\
\hline Small-group discussions & & & $\sqrt{ }$ & & \\
\hline Mini case presentations & & & $\sqrt{ }$ & & \\
\hline Practical engagement in protocol development and implementation & & & $\sqrt{ }$ & & \\
\hline Local leaders in opinion & & & $\sqrt{ }$ & $\sqrt{ }$ & \\
\hline Identifying and engaging resistant staff & & $\sqrt{ }$ & $\sqrt{ }$ & & \\
\hline
\end{tabular}

which the WG identified would be required to effectively engage with each stakeholder group.

\section{Supporting changed practice}

The WG developed three best evidence-based "how to do it" protocols to underpin the selected stroke guideline recommendations:

1. referral to acute rehabilitation of stroke (for secondary medical stakeholders);

2. exercise intensity to improve mobility (for physical therapists);

3. dysphagia screening and pressure ulcer prevention and management (for primary [physiatrists, nurses, speech therapists] and secondary stakeholders).

The protocols provide practical details and supports to assist health care providers, wherever they are located in the Philippines, to implement the selected guideline recommendations. These protocols dovetail with the PARM context points ${ }^{14}$ and ensure the applicability and generalizability of the contextualized guidelines across the Philippines. The WG believed that standard descriptors of assessments and measures, interventions, and timeframes for interventions would assist in not only educating stakeholders about "how to do" the evidence-based recommendations, but would also improve guideline compliance by breaking down the barriers of lack of knowledge and access to resources, as well as by underpinning standard recording of the items required in the audit. An example of a protocol developed from this process is provided in Table S1.

\section{Sample size calculation}

Assuming that the national stroke prevalence is 486 per 100,000 population and an average behavior change of at least $4.5 \%$ could be expected from the strategies used, the total sample size was determined to be 196 (98 for both baseline and audit, equally divided among the four hospitals) with an allocation of ratio of 1 between baseline and audit. ${ }^{25}$ For testing the mean increase between two independent groups, null hypothesis statistical testing was set at $P<0.05$ and power at $\pi=0.80$.

\section{Auditing practice}

The WG discussed how the proposed "current practice" audit would occur. This would be developed as a specific audit protocol. The first stage will be conducted as a descriptive retrospective record audit describing current stroke practices in the Philippines (using the current practice data items in Table 2). Audited patients' records will be from stroke patients from January to December 2012. Medical charts will be reviewed consecutively starting from December 2012 and proceeding backwards until the sample size will be met (25 per institution). Data extraction from these records will be undertaken by local medical interns, physiotherapy, and occupational therapy students at each hospital, who do not have a vested interest in the project outcome. After guideline rollout, a second (re-audit) will be conducted to provide data for a practice improvement activity (using the data in the first shaded column in Table 2). Guideline rollout will be from June to December 2013 and re-audit will commence in August 2013. Table 2 outlines current practice measures of eight demographic items, nine medical history items, two early mobilization items, one increased exercise tolerance item, three discharge summary items, and one functional assessment item.

On re-audit, the group proposed eleven general items in the acute rehabilitation phase for stroke patients. For the special assessments for pressure ulcer and dysphagia, all five items collected initially will be re-audited. Change in key outcome measures attributed to the implementation of the stroke clinical guideline recommendations will be reported 

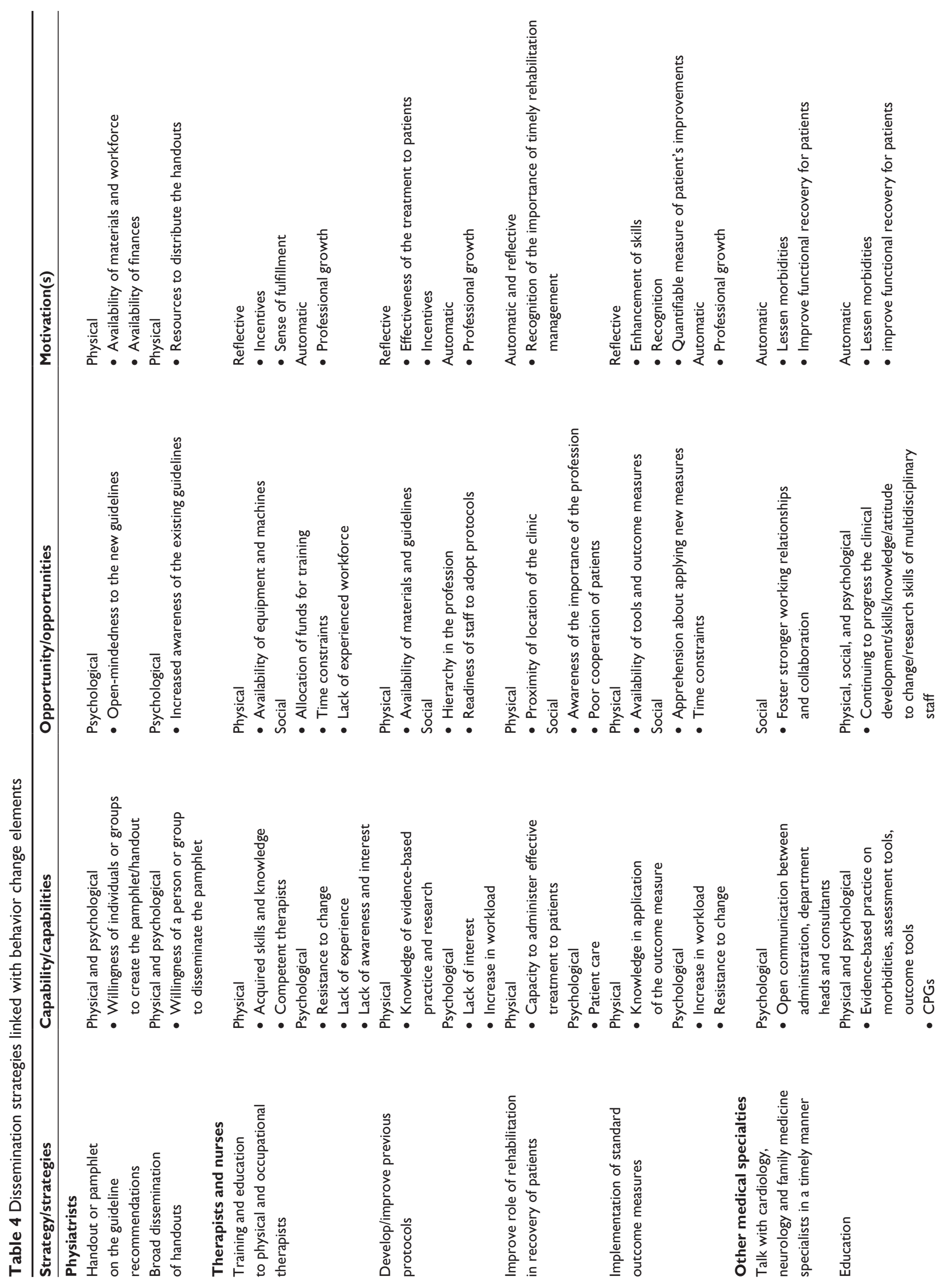

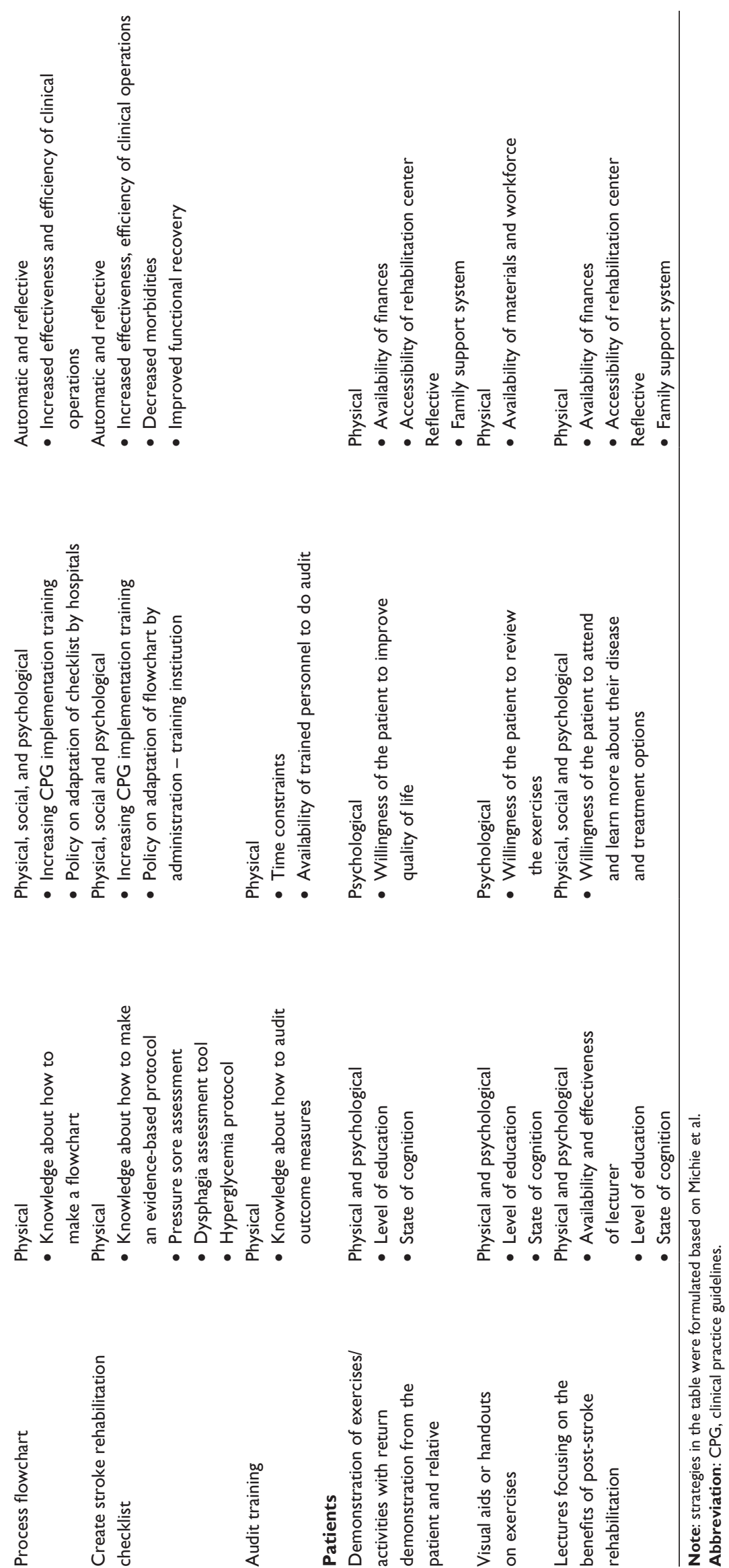
using the parameters in the second shaded column in Table 2. The number of items for re-audit will be kept to a minimum to assist in compliance with data recording and the efficiency of the audit process itself, and in targeting educational interventions to improve record keeping. ${ }^{26}$

In summary, the audit will be a nationwide study involving hospitals that have rehabilitation centers. It will provide a comprehensive profile of stroke patients who are referred for rehabilitation that will include basic information about the patients' demographics, stroke descriptors, the use of outcome measures important in rehabilitation, such as the National Institute of Health Stroke Severity Scale, Barthel index, modified Rankin Scale, the presence of medical comorbidities and rehabilitation complications, early mobilization of patients, pressure sore assessment and management, and dysphagia assessment. It will be able to identify gaps in the recording of important information on current practice, as well as identify areas where records could be improved and used for future quality improvement activities.

Aside from the audit, a questionnaire will be given to those who were involved in the implementation to extract which of the dissemination strategies were effective in improving self-perceived knowledge and change in practice behavior.

The results of the audit will be presented at PARM's national convention, which is held annually, and will be provided to the Department of Health and Philippine Health Insurance Company, which are government agencies responsible for improving the health care delivery system through the implementation of clinical practice guidelines. ${ }^{4}$

\section{Discussion}

This paper presents the protocol to underpin the second stage of PARM's plan to operationalize the implementation of clinical practice guidelines for inpatient stroke rehabilitation. The guideline implementation plan will be rolled out after a current practice audit has been completed to provide a baseline against which practice changes can be compared. The first stage of our work, contextualizing existing Western clinical practice guidelines for Filipino conditions, consumed 12 months of voluntary activity from PARM members during 2011/2012. This involved taking 21 steps, covering initial training; establishing the framework within which recommendations were contextualized; guideline searching; critiquing; and inclusion, contextualization, review, and implementation planning. A writing guide was developed to assist in endorsing recommendations in a standard manner, relevant to a "typical" patient journey, and to contextualize recommendations for local settings. ${ }^{14}$ There was general endorsement from PARM members for the contextualized guidelines in February 2012 at a national convention, and their likelihood of influencing Filipino practices was determined using an electronic survey.

The implementation protocol described in this paper was informed by principles of quality improvement, current best evidence regarding evidence implementation, and models of behavior change. ${ }^{8-12,27}$ Audit and feedback are the most objective assessments of quality improvement and behavior in practice. ${ }^{15}$ Thus, in this project, we took the fundamental step of describing current practice by means of an audit protocol. ${ }^{22}$ It was crucial to undertake this process because country-by-country variations exist in health practices and health systems. ${ }^{9,11}$ The outcomes identified in the audit protocol will also be the basis for assessing changes in practice and behavior after the guideline implementation stage.

In this project, the first step of describing current practice in the Philippines was taken by means of a standard national audit protocol. There was no intention at this stage to compare practices with those of other countries. Many Western countries have put significant investment into establishing safe practice criteria in health care. ${ }^{28}$ However, such improvements can only be mounted once current practice has been established. Transplanting knowledge from developed countries into developing countries without an understanding of country-specific contexts is fraught with danger, as noted by Ramani ${ }^{29}$ and Peabody et al. ${ }^{30}$ Thus, it was essential that Filipino practices be described and understood before widespread practice change could be implemented or comparisons made with quality and safety performance in other countries.

The PARM implementation plan is underpinned by six evidence-based strategies in disseminating the guidelines in the Philippines. These are: (1) designing multifaceted interventions rather than single component interventions; (2) tailoring the interventions to the population; (3) establishing interprofessional and organizational collaboration (collaboration between PARM and the International Centre for Allied Health Evidence, University of South Australia); (4) use of local opinion leaders; (5) the provision of organizational and administrative support; and (6) the identification of stakeholders and their needs, and addressing specific behaviors that could influence guideline uptake. To facilitate all this, significant voluntary effort was provided by the eleven participants, not only over the intensive weekend described here, but also in future ongoing commitments to provide educational and motivational support for peers. 
The implementation plan was based on Western research regarding best-practice effective dissemination strategies for clinical guidelines and measurement of their impact. Michie et al's behavior change model ${ }^{8}$ assisted the WG in considering the different behavioral aspects that needed to be addressed. It was clear when considering behavior change using this model, that a "one size fits all" implementation plan would not address the needs of all stakeholders. Seeing the efficiencies underpinning the PARM implementation plans with current best evidence on behavior change was another learning opportunity for the WG, which agreed that without access to the published implementation science research, its deliberations would have been less well directed and efficient.

The success of the implementation of the stroke guideline requires input not just from physiatrists and therapists but also input and buy in from other medical personnel, nurses, and patients. Timely referral to rehabilitation is required from the secondary medical stakeholders named in the rehabilitation guidelines to ensure that all patients have the opportunity to engage with best-practice rehabilitation, which should commence as early as possible. Nurses' engagement is also essential to support physiatrists' rehabilitation instructions and ensure that patients are offered access to best-practice rehabilitation care, in conjunction with therapists. Patients and their families are important participants, as their willingness to engage with best-practice care and comply with rehabilitation instructions will optimize rehabilitation outcomes. Thus, the engagement of all stakeholders is essential to ensure that the rehabilitation guidelines have credibility and are seen as an integral part of the good stroke care continuum. ${ }^{31}$

\section{Conclusion}

The PARM implementation plan is structured on bestpractice principles of quality improvement, behavior change, and guideline dissemination. By adopting the most effective implementation and dissemination strategies within local contexts to address stakeholders, their relationships within stroke care, and their behaviors regarding adopting evidence-based practice, it is anticipated that the potential for the guidelines to improve stroke care and health outcomes will be optimized.

\section{Authors' contributions}

CBG-S conceptualized and designed the paper, facilitated the workshop, participated in the acquisition of data, drafting and revising the manuscript, and approved the final version to be published. JMRD lectured on the audit process and participated in the acquisition of data and revising of the manuscript. KG conceptualized and designed the paper, lectured on the implementation process, drafted and revised the paper. CBG-S, MSE, EDU, LASL, A-RDM, VM, MERT, CRS-V, JLS, KCS, and PJBF participated in the workshop, provided the protocols and steps necessary in implementing the guidelines, created the flowcharts, and helped in the revision of the manuscript. JBSR and RMM participated in the acquisition of data and revising the manuscript. All the authors read and approved the final manuscript.

\section{Acknowledgments}

The authors thank PARM for supporting the project.

\section{Disclosure}

Funding for this study was received from PARM. The authors declare no other conflicts of interest in this work.

\section{References}

1. Middleton S, McElduff P, Ward J, et al; QASC Trialists Group. Implementation of evidence-based treatment protocols to manage fever, hyperglycaemia, and swallowing dysfunction in acute stroke (QASC): a cluster randomised controlled trial. Lancet. 2011;378(9804): 1699-1706.

2. Ilett PA, Brock KA, Graven CJ, Cotton SM. Selecting patients for rehabilitation after acute stroke: are there variations in practice? Arch Phys Med Rehabil. 2010;91(5):788-793.

3. Navarro J. Prevalence of stroke: a community survey. Phil J Neurol. 2005;9(2):11-15.

4. Andersson B, Alcantara MO. Technical Assistance to the Republic of Philippines for the Health Sector Development Program: ADB TA 4647-PHI; Final Report. Mandaluyong City: Asian Development Bank; 2007. Available from: http://www2.adb.org/Documents/ Reports/Consultant/39066-PHI/39066-PHI-TACR.pdf. Accessed March 15, 2012.

5. World Health Organization. Countries: Philippines. 2012. Available from: http://www.who.int/countries/Phl/en/. Accessed March 15, 2012.

6. Dizon JM, Grimmer-Somers K, Kumar S. A pilot study of the evidence based practice training program for Filipino physiotherapists: emerging evidence on outcomes and acceptability. Internet Journal of Allied Health Sciences and Practice. 2012;10(2). Available from: http://ijahsp. nova.edu/articles/Vol10Num2/Dizon.htm. Accessed June 12, 2013.

7. Gonzalez-Suarez C, Grimmer-Somers K, King E, et al. Contextualizing Western guidelines for stroke and low back pain to a developing country (Philippines): an innovative approach to putting evidence into practice efficiently. Journal of Healthcare Leadership. 2012;4: $141-156$.

8. Michie S, van Stralen M, West R. The behaviour change wheel: a new method for characterising and designing behaviour change interventions. Implement Sci. 2011;6:42.

9. Grol R, Grimshaw J. Evidence-based implementation of evidence-based medicine. Jt Comm J Qual Improv. 1999;25(10):503-513.

10. Greenhalgh T, Robert G, Bate P, Macfarlane F, Kyriakidou O. Diffusion of Innovations in Health Service Organisations: A Systematic Literature Review. Malden, MA: Blackwell; 2005.

11. Grol R, Grimshaw J. From best evidence to best practice: effective implementation of change in patients' care. Lancet. 2003;362(9391): 1225-1230.

12. Grol R, Wensing M, Eccles M. Improving Patient Care: The Implementation of Change in Clinical Practice. Edinburgh: Elsevier; 2005. 
13. Grol $\mathrm{R}$, Wensing $\mathrm{M}$. What drives change? Barriers to and incentives for achieving evidence-based practice. Med J Aust. 2004;180(Suppl 6): S57-S60.

14. Philippine Academy of Rehabilitation Medicine (PARM). Stroke Rehabilitation Guideline. Quezon City: PARM; nd. Available from: http://www.eparm.org/images/STROKE-Guideline.pdf. Accessed January 12, 2012.

15. Ivers $\mathrm{N}$, Jamtvedt $\mathrm{G}$, Flottorp $\mathrm{S}$, et al. Audit and feedback: effects on professional practice and healthcare outcomes. Cochrane Database Syst Rev. 2012;6:CD000259.

16. Forsetlund L, Bjørndal A, Rashidian A, et al. Continuing education meetings and workshops: effects on professional practice and health care outcomes. Cochrane Database Syst Rev. 2009;(2):CD003030.

17. O'Brien MA, Rogers S, Jamtvedt G, et al. Educational outreach visits: effects on professional practice and health care outcomes. Cochrane Database Syst Rev. 2007;(4):CD000409.

18. Baker R, Camosso-Stefinovic J, Gillies C, et al. Tailored interventions to overcome identified barriers to change: effects on professional practice and health care outcomes. Cochrane Database Syst Rev. 2010;(3):CD005470.

19. Flodgren G, Parmelli E, Doumit G, et al. Local opinion leaders: effects on professional practice and health care outcomes. Cochrane Database Syst Rev. 2011;(8):CD000125.

20. Grimshaw JM, Thomas RE, MacLennan G, et al. Effectiveness and efficiency of guideline dissemination and implementation strategies. Health Technol Assess. 2004;8(6):iii-iv, 1-72.

21. Zwarenstein M, Goldman J, Reeves S. Interprofessional collaboration: effects of practice-based interventions on professional practice and healthcare outcomes. Cochrane Database Syst Rev. 2009;(3): CD000072.

22. Campbell SM, Braspenning J, Hutchinson A, Marshall M. Research methods used in developing and applying quality indicators in primary care. Qual Saf Health Care. 2002;11(4):358-364.

23. Davies H. Measuring and Reporting the Quality of Health Care: Issues and Evidence from the International Research Literature. Edinburgh: NHS Quality Improvement Scotland; 2005. Available at: http://www.healthcareimprovementscotland.org/his/idoc. ash $x$ ?docid=d55b6ead-745b-44e7-8fcc-8f3e379da22b\&version=-1 . Accessed June 12, 2013.
24. Australian Institute of Health and Welfare (AIHW). Towards National Indicators of Safety and Quality in Health Care. Canberra: AIHW; 2009. Available from: http://www.aihw.gov.au/WorkArea/DownloadAsset. aspx?id=6442457172. Accessed May 24, 2013.

25. Grimshaw JM, Eccles MP, Lavis JN, Hill SJ, Squires JE. Knowledge translation of research findings. Implement Sci. 2012;7:50.

26. Strating MMH, Nieboer AP, Zuiderent-Jerak T, Bal RA. Creating effective quality-improvement collaboratives: a multiple case stud. BMJ Qual Saf. 2011;20:344-350.

27. Michie S, Johnston M, Abraham C, Lawton R, Parker D, Walker A; "Psychological Theory" Group. Making psychological theory useful for implementing evidence based practice: a consensus approach. Qual Saf Health Care. 2005;14(1):26-33.

28. AIHW. International information on safety and quality of health care [web page on the Internet]. Canberra: AIHW; nd. Available from: http://www.aihw.gov.au/sqhc-international-information/. Accessed June 13, 2013.

29. Ramani S. Can we transplant conceptual frameworks of healthcare quality evaluation from developed countries into developing countries? Indian J Community Med. 2009;34(2):87-88.

30. Peabody JW, Taguiwalo MM, Robalino DA, Frenk J. Improving the quality of health care in developing countries. In: Jamison DT, Breman JG, Measham AR, Alleyne G, Claeson M, editors. Disease Control Priorities in Developing Countries. 2nd ed. Washington DC: World Bank; 2006:1293-1307.

31. Hamilton S, McLaren S, Mulhall A. Assessing organisational readiness for change: use of diagnostic analysis prior to the implementation of a multidisciplinary assessment for acute stroke care. Implement Sci. 2007;2:21. 


\section{Supplementary material}

Table SI Protocol for referral for acute stroke rehabilitation

A. Medical assessment performed by internist - neurologist/ cardiologist/family medicine physician

I. Any age, diagnosed to have first or recurrent stroke, admitted within 24 hours

2. Systolic blood pressure between 120 and $220 \mathrm{mmHg}$

3. Oxygen saturation of $92 \%$ (with or without supplementation)

4. Heart rate between 40 and 100 beats per minute

5. Temperature $<38.5^{\circ} \mathrm{C}$

B. Contraindications to acute stroke rehabilitation'

I. Deterioration within the first hour of admission to the stroke unit or direct admission to intensive care

2. Concurrent progressive neurologic disorder

3. Acute coronary syndrome

4. Severe heart failure

C. Rehabilitation medicine assessment ${ }^{2}$

A. Initial brief assessment

I. Risk factors for stroke recurrence: uncontrolled diabetes mellitus and blood pressure; increased cholesterol levels

2. Presence of medical comorbidities that may affect rehabilitation

3. Level of consciousness

4. Cognitive status

5. Assessment of swallowing impairment

6. Assessment of risk in developing pressure sores

7. Impairment in mobility

8. Assessment of risk in developing deep vein thrombosis

B. Rehabilitation needs assessment

I. Prevention of possible complications due to stroke and immobilization
a. Dysphagia
b. Pressure ulcers
c. Risk of deep venous thrombosis
d. Bowel and bladder dysfunction
e. Presence of pain
f. Malnutrition

2. Assessment of impairments

a. Cognitive impairment

b. Communication deficits

c. Motor impairment in affected extremities

d. Sensory deficit in affected extremities

e. Visuospatial impairment

f. Disturbance like depression or anxiety

3. Psychosocial assessment and presence of family support

4. Functional outcome measures using Functional Independent

Measure Scale

\section{References}

1. Bernhardt J, Dewey H, Thrift A, Collier J, Donnan G. A very early rehabilitation trial for stroke (AVERT): phase II safety and feasibility. Stroke. 2008;39(2):390-396.

2. Duncan PW, Zorowitz R, Bates B, et al. Management of Adult Stroke Rehabilitation Care: a clinical practice guideline. Stroke. 2005;36(9): e100-e143.
Clinical Audit

\section{Publish your work in this journal}

Clinical Audit is an international, peer-reviewed, open access journal focusing on the processes and outcomes of clinical audit in any area of healthcare. All aspects of patient care are addressed within the journal and practitioners from all disciplines are invited to submit their work. Areas covered include: Publication of audits; How an audit has changed practice;

\section{Dovepress}

Practical tips on how to do audits and to avoid pitfalls; How audits have changed patient care; Calls and justifications for new audits. The manuscript management system is completely online and includes a very quick and fair peer-review system, which is all easy to use. Visit http://www.dovepress. com/testimonials.php to read real quotes from published authors. 